Gazi University
Journal of Science
http://dergipark.gov.tr/gujs

\title{
New Parametric Estimation Methods based on Ranked Set Sampling
}

\author{
Samir K. Ashour ${ }^{1}$ (D) , Mohamed S. Abdallah ${ }^{2 *}$ (D) \\ ${ }^{I}$ Cairo University, Institute of Statistical Studies \& Research, Egypt \\ ${ }^{2}$ Aswan University, Faculty of Commerce, Egypt
}

\section{Highlights}

- We show how missing data approach can be useful for introducing new parametric estimation methods.

- EM algorithm and linear interpolation technique were used under ranked set sampling.

- Fraction-of-random rankings model was used, for the first time, for deriving new parametric method.

- It was shown numerically that the proposed methods were superior to all their competitors.

\section{Article Info}

Received: $02 / 06 / 2018$ Accepted: 13/11/2018

\begin{tabular}{l} 
Keywords \\
\hline Cramér-von-Mises \\
EM algorithm \\
Estimation methods \\
Missing Data Approach \\
Ranked set sampling
\end{tabular}

\section{INTRODUCTION}

The problem of parameters estimation occupied an important position in various areas of researches such as agricultural, biological, ecological, engineering, medical, physical, and social sciences. As parameters estimation can be considered as an essential step during performing many statistical aspects (e.g. testing hypotheses, goodness of fit, reliability analysis, ... etc.). With this motivation, many efforts are devoted to produce several estimation methods which are available in the literature. However, the appeal of the estimation methods vary from researcher to another. For instance, one may prefer to use the moment estimators (MEs) rather than the maximum likelihood estimators (MLEs) in the case that the parent distribution is a more complicated leading the computations may not converge. For more discussion about different types of methods estimation, one can refer to Mittelhammer [1].

In the real life, there are several situations that the variable of interest $Y$ can be more easily ranked by either visual inspection or using a concomitant variable $X$ than quantified, ranked set sample (RSS) introduced by McIntyre [2] is widely recommended to be adopted in such these situations. The attractive property of RSS is it provides an informative image of the population in a more efficient way than other traditional sampling techniques such as simple random sample (SRS). The reason is that RSS depends on the information supported by not only the exact quantification of the $Y$ 's items but also their rankings. The basic procedures of concomitant-based RSS can be briefly described as follows:

First, draw $k$ random samples each of size $k$ of the bivariate variables $(X, Y)$. Second, for each sample, $X$ values are fully measured and sorted in ascending way. Then, measure only the $Y$ values associated with the $i^{\text {th }}$ smallest observation of $X$ corresponding to the $i^{\text {th }}$ sample $(i=1 \ldots k)$. Finally, repeat the preceding 
steps $m$ times (cycles) in order to obtain $n=k m$ values of $Y$. If the number of the selected items across the cycles equals, RSS is hence called balanced RSS. Otherwise, it is called unbalanced RSS. It should be emphasized that we will confine ourselves that $(X, Y)$ are continuous variables drawn through balanced RSS. In order to ease the notation, let $x_{l j(i)}$ be the $i^{\text {th }}$ smallest observation from $j^{\text {th }}$ sample corresponding to $l^{\text {th }}$ cycle. And also, let $y_{l i[i]}$ be the interested variable's values associated with $x_{l j(i)}((i, j)=1 . . k, l=$ $1 \ldots m$ ). Further, Perfect (Imperfect) ranking refers that the rankings are done without (with) errors denoted by rounded (squared) bracket.

Although RSS actually is a nonparametric in its nature, a large number of publications for parametric estimation has been produced in the literature. From those, Abu-Dayyeh et al. [3] used different estimation methods for estimating the shape and scale parameters of Pareto distribution based on RSS and SRS. Sarikavanij et al. [4] made a comparison study between SRS and RSS for estimating the location and scale parameters of a two-parameter exponential distribution. Hassan [5] did a study about exponentiated exponential distribution using both SRS and RSS techniques based on maximum likelihood and Bayesian estimation methods. Yousef and Al-Subh [6] compared between the efficiency of RSS and SRS in estimating the location and scale parameters of the Gumbel distribution using different estimation method. Dey et al. [7] addressed the estimation of the parameter of Rayleigh distribution using different methods of frequentist and Bayesian estimation approaches based on SRS and RSS and other sampling schemes. For other interesting works related to RSS, see Arslan and Ozturk [8].

It is well established by many publications that the superiority of RSS depends heavily on the quality of ranking information. As this superiority increases (decreases) in the case of perfect (imperfect) ranking. Therefore it is expected that the statistical procedures which assume the perfect assumption have a poor performance when this assumption is violated. One way to overcome this problem is to construct such these statistical procedures under imperfect ranking models. This motivated us to derive parametric methods for estimating the location and scale parameters which implicitly use the stochastic order property and be less sensitive to the departure of the perfect ranking as well. In this article, three novel methods of estimation of the parameters are proposed. The first one is derived under Fraction-of-random rankings model, the others are constructed based on Pool-Adjacent-Violators Algorithm (PAVA).

The remainder of this manuscript is structured as follows. Section 2 exhibits several popular methods of parameter estimation. Section 3 presents the three proposed estimation methods. The numerical comparisons using Monte Carlo simulation are summarized and organized in Section 4. In Section 5, real data set is used to present the applicability of the estimation methods, and lastly Section 6 shows our concluding points and some possible extension works.

\section{THE ESTIMATION METHODS}

Let $\left\{y_{l i[i]}: i=1 \ldots k ; l=1 \ldots m\right\}$ be a concomitant-based RSS of size $n$ drawn from a location and scale family of distribution $F\left(\frac{y-\mathcal{M}}{\delta}\right)$, where $F(\cdot)$ is the cumulative distribution function (CDF) having mean zero and scale 1 of the standardized random variable $\frac{Y-\mathcal{M}}{\delta}$, and the support of $F(\cdot)$ does not depend on $\theta=$ $(\mathcal{M}, \delta)$. Although several methods are available to estimate $\theta$, there is no universal agreement about the most efficient method. In this part, four estimation procedures are briefly discussed one by one as follows.

\subsection{Moments Estimators}

This method is firstly used by Karl Pearson (see Rajagopalan and Dhanavanthan [9]) which is based on the well-known fact that the population moments are functions of the unknown parameters and because of the consistency of the sample moments to the population moments, this method works through solving a system of equations constructing by equating the first $M$ raw moments for the population distribution with their correspomding raw sampling moments. Mathematically, the system of equations can be expressed as: 


$$
E\left(Y^{r}\right)=\hat{\mu}^{r}=\frac{1}{n} \sum_{i=1}^{k} \sum_{l=1}^{m} y_{l i[i]}^{r} \quad r=1,2 \ldots M,
$$

where $M$ is the number of the unknown parameters. Here $M=2$ and the estimates obtained by solving (1) will be denoted as $\hat{\theta}_{M}=\left(\widehat{\mathcal{M}}_{M}, \hat{\delta}_{M}\right)$.

\subsection{Maximum Likelihood Estimators}

Stokes [10] utilized the fact the items of RSS are, under the perfectness, a random sample of $i^{\text {th }}$ order statistics of size $k$ with alternatively likelihood function given by:

$$
\begin{aligned}
L_{S}(\mathcal{M}, \delta \mid Y)= & \sum_{l=1}^{m} \sum_{i=1}^{k} \log \left(f_{i}\left(\frac{y_{l i[i]}-\mathcal{M}}{\delta}\right)\right) \propto-n \log (\delta)+\sum_{l=1}^{m} \sum_{i=1}^{k} \log \left(f\left(\frac{y_{l i[i]}-\mathcal{M}}{\delta}\right)\right) \\
& +\sum_{l=1}^{m} \sum_{i=1}^{k}(i-1) \log \left(F\left(\frac{y_{l i[i]}-\mathcal{M}}{\delta}\right)\right)+\sum_{l=1}^{m} \sum_{i=1}^{k}(k-i) \log \left(1-F\left(\frac{y_{l i[i]}-\mathcal{M}}{\delta}\right)\right),
\end{aligned}
$$

where $f_{i}(\cdot)$ is the probability density function (pdf) of $i^{t h}$ order statistics. The MLE of $\mathcal{M}$ and $\delta$ can be obtained by either maximizing $L_{S}(\mathcal{M}, \delta \mid Y)$ or solving simultaneously the following two equations:

$$
\left.\begin{array}{c}
\frac{\partial L_{S}(\mathcal{M}, \delta \mid Y)}{\partial \mathcal{M}}=\sum_{l=1}^{m} \sum_{i=1}^{k} \frac{f_{i}^{\prime \mathcal{M}}\left(\frac{y_{l i[i]}-\mathcal{M}}{\delta}\right)}{f_{i}\left(\frac{y_{l i[i]}-\mathcal{M}}{\delta}\right)}=0 \\
\frac{\partial L_{S}(\mathcal{M}, \delta \mid Y)}{\partial \delta}=\sum_{l=1}^{m} \sum_{i=1}^{k} \frac{f_{i}^{\prime} \delta\left(\frac{y_{l i[i]}-\mathcal{M}}{\delta}\right)}{f_{i}\left(\frac{y_{l i[i]}-\mathcal{M}}{\delta}\right)}=0
\end{array}\right\}
$$

where $f^{\prime a}(\cdot)$ is the first derivative of $f(\cdot)$ with respect to $a$. The corresponding estimates of solving (2) will be denoted as $\hat{\theta}_{L}=\left(\widehat{\mathcal{M}}_{L}, \hat{\delta}_{L}\right)$.

\subsection{Cramér-von-Mises Estimators}

Another class of parameters estimation method can be obtained by using the well-known goodness of fit statistics such as Kolmogorov-Smirnov, Cramér-von Mises and Anderson-Darling statistics. The idea is to select the estimates that minimize the distance between the sampling distribution function and the parent CDF. Interestingly, Macdonald [11] provided empirical evidence that the bias of the estimates based on Cramér-von Mises is smaller than those based on the other goodness of fit statistics. This motivated us to adopt Cramér-von Mises statistic which can be expressed as:

$$
L_{C}^{*}(\mathcal{M}, \delta \mid Y)=n \int_{-\infty}^{\infty}\left(F\left(\frac{y-\mathcal{M}}{\delta}\right)-\hat{F}(y)\right)^{2} d F\left(\frac{y-\mathcal{M}}{\delta}\right)
$$

where $\hat{F}(y)$ is the sampling CDF frequentely be estimated by the empirical distribution function (EDF) leading (3) takes a simpler formula given by:

$$
L_{C}(\mathcal{M}, \delta \mid Y)=\frac{1}{12 n}+\frac{1}{n} \sum_{i=1}^{n}\left(F\left(\frac{y_{(i)}^{*}-\mathcal{M}}{\delta}\right)-\frac{(2 i-1)}{2 n}\right)^{2}
$$

where $y_{i}^{*}$ 's are the pooled values of $y_{l[i]}$ 's across all the cycles, $y_{(i)}^{*}$ 's are ordered values of $y_{i}^{*}$ 's. Hence Cramér-von Mises estimators can be obtained either by minimizing $L_{C}(\mathcal{M}, \delta \mid Y)$ or equivalently solving simultaneously the following equations: 


$$
\begin{aligned}
& \frac{\partial L_{C}(\mathcal{M}, \delta \mid Y)}{\partial \mathcal{M}}=\sum_{i=1}^{n}\left(F\left(\frac{y_{(i)}^{*}-\mathcal{M}}{\delta}\right)-\frac{(2 i-1)}{2 n}\right) F^{\prime \mathcal{M}}\left(\frac{y_{(i)}^{*}-\mathcal{M}}{\delta}\right)=0 \\
& \frac{\partial L_{C}(\mathcal{M}, \delta \mid \mathrm{Y})}{\partial \delta}=\sum_{i=1}^{n}\left(F\left(\frac{y_{(i)}^{*}-\mathcal{M}}{\delta}\right)-\frac{(2 i-1)}{2 n}\right) F^{\prime \delta}\left(\frac{y_{(i)}^{*}-\mathcal{M}}{\delta}\right)=0
\end{aligned}
$$

The Cramér-von Mises estimators will be denoted as $\hat{\theta}_{C}=\left(\widehat{\mathcal{M}}_{C}, \hat{\delta}_{C}\right)$. For further interesting properties about Cramér-von Mises estimators, reader can refer to Ozturk and Hettmansperger [12].

\subsection{Maximum Product Of Spacings Estimators}

Mahdizadeh and Tamandi [13] suggested recently to adopt the maximum product of spacing (MPS) Method, proposed by Cheng and Amin [14], for getting the parameter estimates rather than using MLE approach proposed by Stokes [10] under RSS. MPS is based on the idea of expressing the likelihood function by differences between the values of the CDF at consecutive data points which can be expressed as:

$$
L_{T}(\mathcal{M}, \delta \mid Y)=\sum_{i=1}^{n+1} \log \left(F\left(\frac{y_{(i)}^{*}-\mathcal{M}}{\delta}\right)-F\left(\frac{y_{(i-1)}^{*}-\mathcal{M}}{\delta}\right)\right)
$$

where $F\left(\frac{y_{(0)}^{*}-\mathcal{M}}{\delta}\right)=0$ and $F\left(\frac{y_{(n+1)}^{*}-\mathcal{M}}{\delta}\right)=1$. Hence the estimates of $\theta=(\mathcal{M}, \delta)$ can be obtained either by maximizing $L_{T}(\mathcal{M}, \delta \mid Y)$ or equivalently solving simultaneously the equations in (4):

$$
\left.\begin{array}{l}
\frac{\partial L_{T}(\mathcal{M}, \delta \mid Y)}{\partial \mathcal{M}}=\sum_{i=1}^{n+1} \frac{F^{\prime \mathcal{M}}\left(\frac{y_{(i)^{*}}^{*}-\mathcal{M}}{\delta}\right)-F^{\prime \mathcal{M}}\left(\frac{y_{(i-1)}^{*}-\mathcal{M}}{\delta}\right)}{F\left(\frac{y_{(i)}^{*}-\mathcal{M}}{\delta}\right)-F\left(\frac{y_{(i-1)^{-\mathcal{M}}}^{*}}{\delta}\right)}=0 \\
\frac{\partial L_{T}(\mathcal{M}, \delta \mid Y)}{\partial \delta}=\sum_{i=1}^{n+1} \frac{F^{\prime \delta}\left(\frac{y_{(i)}^{*}-\mathcal{M}}{\delta}\right)-F^{\prime \delta}\left(\frac{\left.y_{(i-1)}^{*}\right)^{\mathcal{M}}}{\delta}\right)}{F\left(\frac{y_{(i)^{-\mathcal{M}}}^{*}}{\delta}\right)-F\left(\frac{y_{(i-1)^{*}-\mathcal{M}}^{*}}{\delta}\right)}=0
\end{array}\right\} .
$$

The corresponding estimates of $\theta=(\mathcal{M}, \delta)$ for MPS method will be denoted as $\hat{\theta}_{T}=\left(\widehat{\mathcal{M}}_{T}, \hat{\delta}_{T}\right)$.

\section{THE PROPOSED ESTIMATION METHODS}

In this part three novel parametric methods of estimation under RSS are suggested and presented. Our idea is to incorporate the information supported by the unmeasured items together with the measured items for suggesting new parametric method as shown below.

\subsection{Iterative Moments Estimators}

Our first proposed estimation method is based on incorporating the stochastic relationship between the measured items and the unmeasured items to estimate $\theta=(\mathcal{M}, \delta)$. In more precise, our strategy is to impute the unmeasured items in the view of the measured items, then estimate $\theta=(\mathcal{M}, \delta)$ using $(1)$ based on the information provided by both the measured items and the imputed unmeasured items, finally use the parameters' estimates to upgrade these imputed unmeasured items. By repeating these steps iteratively until the convergence of the parameters' estimates occurs. Imputing the unmeasured items will be achieved in a parametric fashion using the conditional order statistics pdf as explained below:

- If $y_{l j[i]}>y_{l i[i]}$, then: 


$$
E\left(y^{r} \mid y>y_{l i[i]}\right)=\int_{y_{l i[i]}}^{\infty} y^{r} f_{j, i}^{1}\left(y, \mathcal{M}, \delta \mid y_{l i[i]}\right) d y
$$

- In contradiction, if $y_{l j[i]}<y_{l i[i]}$, then:

$$
E\left(y^{r} \mid y<y_{l i[i]}\right)=\int_{-\infty}^{y_{l i[i]}} y^{r} f_{j, i}^{2}\left(y, \mathcal{M}, \delta \mid y_{l i[i]}\right) d y .
$$

where $f_{j, i}^{1}\left(y \mid y_{l i[i]}\right)$ and $f_{j, i}^{2}\left(y \mid y_{l i[i]}\right)$ are the conditional order statistics pdfs respectively expressed as:

$$
\begin{aligned}
& f_{j, i}^{1}(y \mid \mathrm{w})= \\
& \frac{(k-i) !}{(j-i-1) !(k-j) !}\left(\frac{F(y, \mathcal{M}, \delta)-F(\mathrm{w}, \mathcal{M}, \delta)}{1-F(w, \mathcal{M}, \delta)}\right)^{j-i-1}\left(1-\frac{F(y, \mathcal{M}, \delta)-F(w, \mathcal{M}, \delta)}{1-F(w, \mathcal{M}, \delta)}\right)^{k-j} \frac{f(y, \mathcal{M}, \delta)}{1-F(w, \mathcal{M}, \delta)} \\
& w \leq y \leq \infty
\end{aligned}
$$

and

$f_{j, i}^{2}(y \mid \mathrm{w})=\frac{(i-1) !}{(j-1) !(i-j-1) !}\left(\frac{F(y, \mathcal{M}, \delta)}{F(w, \mathcal{M}, \delta)}\right)^{j-1}\left(1-\frac{F(y, \mathcal{M}, \delta)}{F(w, \mathcal{M}, \delta)}\right)^{i-j-1} \frac{f(y, \mathcal{M}, \delta)}{F(w, \mathcal{M}, \delta)}$,

$$
-\infty \leq y \leq w
$$

By applying (5) and (6) to the entire sample and putting them together, we will get:

$$
\mu_{E}^{r *}(\mathcal{M}, \delta)=\frac{1}{k n} \sum_{l=1}^{m} \sum_{i=1}^{k}\left(y_{l i[i]}^{r}+E\left(y^{r} \mid y>y_{l i[i]}\right)+E\left(y^{r} \mid y<y_{l i[i]}\right)\right) .
$$

It is observant that $\mu_{E}^{r *}(\mathcal{M}, \delta)$ is dependable only if the perfectness assumption is hold. However when this condition is violated, $\mu_{E}^{r *}(\mathcal{M}, \delta)$ may give illogical values. To tackle this dilemma, we resort to use fraction-of-random-rankings model proposed by Frey et al. [15] to modify $\mu_{E}^{r *}(\mathcal{M}, \delta)$ as being more robust to the departure from perfect rankings as presented below:

$$
\mu_{E}^{r}(\mathcal{M}, \delta)=\frac{1}{k n} \sum_{l=1}^{m} \sum_{i=1}^{k}\left((1-\lambda)\left(y_{l i[i]}^{r}+E\left(y^{r} \mid y>y_{l i[i]}\right)+E\left(y^{r} \mid y<y_{l i[i]}\right)\right)+\lambda E\left(y^{r}\right)\right) .
$$

Since $\mu_{E}^{r}(\mathcal{M}, \delta)$ can not be directly computed as it simply relies on the unknown parameters $\mathcal{M}, \delta$ and $\lambda$, EM algorithm is adopted to overcome this problem whose steps are described below.

1- Let $\left(\widehat{\mathcal{M}}^{(0)}, \hat{\delta}^{(0)}\right)$ be the seed estimates for $\mathcal{M}, \delta$.

2- Set $p=0$.

3- Estimate the parameter $\lambda$ by the corresponding likelihood function of fraction-of-random-rankings model (see Frey and Zhang [16] and Ashour and Abdallah [17]):

$$
\hat{\lambda}=\operatorname{Max}_{\lambda \in[0,1]} \prod_{i=1}^{k} \prod_{l=1}^{h}\left((1-\lambda) b_{i, k-i+1}\left(F\left(y_{[i, l]}\right), \widehat{\mathcal{M}}^{(p)}, \hat{\delta}^{(p)}\right)+\lambda\right) .
$$

where $b_{a, b}(t)$ is the pdf of the Beta distribution with parameters $a$ and $b$ at the point $t$.

4- In the light of (5) and (6), compute the sampling raw moments by the following equation:

$$
\hat{\mu}_{E}^{r}\left(\widehat{\mathcal{M}}^{(p)}, \hat{\delta}^{(p)}\right)=\frac{1}{k n} \sum_{l=1}^{m} \sum_{i=1}^{k}\left((1-\hat{\lambda})\left(y_{l i[i]}^{r}+E\left(y^{r} \mid y>y_{l i[i]}\right)+E\left(y^{r} \mid y<y_{l i[i]}\right)\right)+\hat{\lambda} E\left(y^{r}\right)\right) .
$$


5- Use $\hat{\mu}_{E}^{1}(\mathcal{M}, \delta)$ and $\hat{\mu}_{E}^{2}(\mathcal{M}, \delta)$ for estimating $\mathcal{M}, \delta$ by solving the following system:

$$
E\left(Y^{r}\right)=\hat{\mu}_{E}^{r}(\mathcal{M}, \delta) . \quad r=1,2
$$

Denote the solution of (7) by $\left(\widehat{\mathcal{M}}^{(p+1)}, \hat{\delta}^{(p+1)}\right)$.

6- Set $p=p+1$.

7- Repeat steps $(3-6)$ until stopping rule satisfies. i.e.

$$
\operatorname{Max}\left(\left|\widehat{\mathcal{M}}^{(p)}-\widehat{\mathcal{M}}^{(p-1)}\right|,\left|\hat{\delta}^{(p)}-\hat{\delta}^{(p-1)}\right|\right) \leq .001 .
$$

8- The final estimates are $\hat{\theta}_{E}=\left(\widehat{\mathcal{M}}_{E}, \hat{\delta}_{E}\right)=\left(\widehat{\mathcal{M}}^{(p)}, \hat{\delta}^{(p)}\right)$.

Remark 1. In the light of the condition (10) of $\mathrm{Wu}$ [18], the suggested EM algorithm will always converge to a local maximum as long as $F(\cdot)$ belongs to the exponential family. It is hence advisable to adopt $\hat{\theta}_{E}$ in the case of $F(\cdot)$ as a member of the exponential family.

Remark 2. Under the perfectness assumption and in the light of the law of large numbers, one can show that $\hat{\mu}_{E}^{r}\left(\widehat{\mathcal{M}}_{E}, \hat{\delta}_{E}\right)$ is a consistent estimator to $E\left(Y^{r}\right)$ causing $\hat{\theta}_{E}$ to be a consistent estimator to $\theta$.

\subsection{Modified Moments Estimators}

The second modification of $\hat{\theta}_{M}$ consists of replacing $\hat{\mu}^{r}$ in (1) by another more efficient estimator. As opposite to $\hat{\theta}_{E}$, one can also impute the unmeasured items in a nonparametric way using the information ranking supported by $X$. In the light of Zamanzade and Mohammadi [19], $E\left(Y^{r}\right)$ can be estimated through applying the following steps:

1- Combining $y_{l[i]}$ and their corresponding values of $x_{l i(i)}$ into two new variables $\left(y_{z}^{*}, x_{z}^{*}, z=1 \ldots n\right)$ respectively.

2- Sorting ascending $\left(y_{z}^{*}, x_{z}^{*}\right)$ according to $x^{*}$ values yielding $\left(y_{[z]}^{*}, x_{(z)}^{*}\right)$.

3- Computing the $r$ th raw sampling moments as:

$$
\hat{\mu}_{X}^{r}=\frac{1}{n k} \sum_{t=1}^{n k} h_{1}^{r}\left(x_{(t)}^{*}\right)
$$

where

$$
h_{1}^{r}(x)=\left\{\begin{array}{ccc}
y_{[1]}^{r i s o} & x<x_{(1)}^{*} \\
y_{[t]}^{r i s o}+\frac{y_{[t+1]}^{r i s o}-y_{[t]}^{r i s o}}{x_{(t+1)}^{*}-x_{(t)}^{*}}\left(x-x_{(t)}^{*}\right) & x_{(t)}^{*}<x<x_{(t+1)}^{*} & t=1 \ldots n-1 \\
y_{[n]}^{r i s o} & x_{(n)}^{*}<x & . \\
y_{[t]}^{r i s o} & x=x_{(t)}^{*} & t=1 \ldots n
\end{array}\right.
$$

and $y^{\text {riso }}$ is the isotonized values of $y^{r}$ obtained by the PAVA. For more discussion about PAVA, one can refer to Ozturk [20].

By replacing $\hat{\mu}$ with $\hat{\mu}_{X}^{r}$ in (1), we will get that:

$$
E\left(Y^{r}\right)=\hat{\mu}_{X}^{r}=\frac{1}{n k} \sum_{t=1}^{n k} h_{1}^{r}\left(x_{(t)}^{*}\right) . \quad r=1,2
$$


The estimates produced by solving (8) will be denoted as $\hat{\theta}_{X}=\left(\widehat{\mathcal{M}}_{X}, \hat{\delta}_{X}\right)$.

\subsection{Modified Cramér-Von Mises Estimators}

Due to a plethora CDF estimators provided in the literature particularly in the case of RSS, it is quite reasonable to replace $\hat{F}(y)$ with an efficient CDF estimator rather than the EDF in (4). Most recently, Zamanzade and Mahdizadeh [21] suggested a novel CDF estimator based on a concomitant-based RSS. The authors showed numerically that their estimator is at least as efficient as EDF which can be expressed as:

$$
\hat{F}_{X}(t)=\frac{1}{k n} \sum_{i=1}^{k} \sum_{j=1}^{k} \sum_{l=1}^{m} \hat{F}_{x_{l j(i)}}(t)
$$

where

$$
\hat{F}_{x}(t)=\left\{\begin{array}{cc}
\hat{F}_{1}^{i s o}(t) & x \leq x_{(1)}^{*} \\
\hat{F}_{z}^{i s o}(t)+\frac{\hat{F}_{z+1}^{i s o}(t)-\hat{F}_{z}^{i s o}(t)}{x_{(z+1)}^{*}-x_{(z)}^{*}}\left[x-x_{(z)}^{*}\right] & x_{(z)}^{*} \leq x<x_{(z+1)}^{*} \quad z=1 \ldots n-1, \\
\hat{F}_{n}^{i s o}(t) & x \geq x_{(n)}^{*}
\end{array}\right.
$$

where $\hat{F}_{z}^{i s o}(t)$ is the isotonized values of $I\left(y_{[z]}^{*} \leq t\right)$ and $I(\cdot)$ is the indicator function. For a more discussion about $\widehat{F}_{X}(t)$, see Ashour and Abdallah [22]. One can rewrite the Cramér-von Mises statistic into another general formula as:

$$
\begin{aligned}
L_{F}^{*}(\mathcal{M}, \delta \mid Y) & =n \sum_{i=0}^{n} \int_{y_{(i)}^{*}}^{y_{(i+1)}^{*}}\left(F\left(\frac{y-\mathcal{M}}{\delta}\right)-\hat{F}\left(y_{[i]}^{*}\right)\right)^{2} d F\left(\frac{y-\mathcal{M}}{\delta}\right) \\
& =\frac{n}{3} \sum_{i=0}^{n}\left(\left(F\left(\frac{y_{(i+1)}^{*}-\mathcal{M}}{\delta}\right)-\hat{F}\left(y_{(i)}^{*}\right)\right)^{3}-\left(F\left(\frac{y_{(i)}^{*}-\mathcal{M}}{\delta}\right)-\hat{F}\left(y_{(i)}^{*}\right)\right)^{3}\right) .
\end{aligned}
$$

where $y_{(0)}^{*}=-\infty, y_{(n+1)}^{*}=\infty$. By replacing $\hat{F}\left(y_{(i)}^{*}\right)$ with $\widehat{F}_{x}\left(y_{(i)}^{*}\right)$ yields to:

$L_{F}(\mathcal{M}, \delta \mid Y)=\frac{n}{3} \sum_{i=0}^{n}\left(\left(F\left(\frac{y_{(i+1)}^{*}-\mathcal{M}}{\delta}\right)-\hat{F}_{x}\left(y_{(i)}^{*}\right)\right)^{3}-\left(F\left(\frac{y_{(i)}^{*}-\mathcal{M}}{\delta}\right)-\hat{F}_{x}\left(y_{(i)}^{*}\right)\right)^{3}\right)$

Hence the modified Cramér-von Mises estimators can be obtained either by minimizing $L_{F}(\mathcal{M}, \delta \mid Y)$ or equivalently solving simultaneously the following equations:

$$
\left.\begin{array}{l}
\frac{\partial L_{F}(\mathcal{M}, \delta \mid Y)}{\partial \mathcal{M}}=\sum_{i=0}^{n}\left(\left(F\left(\frac{y_{(i+1)}^{*}-\mathcal{M}}{\delta}\right)-\hat{F}_{x}\left(y_{(i)}^{*}\right)\right)^{2} F^{\prime \mathcal{M}}\left(\frac{y_{(i+1)}^{*}-\mathcal{M}}{\delta}\right)-\left(F\left(\frac{y_{(i)}^{*}-\mathcal{M}}{\delta}\right)-\hat{F}_{x}\left(y_{(i)}^{*}\right)\right)^{2} F^{\prime \mathcal{M}}\left(\frac{y_{(i)}^{*}-\mathcal{M}}{\delta}\right)\right)=0 \\
\frac{\partial L_{F}(\mathcal{M}, \delta \mid Y)}{\partial \delta}=\sum_{i=0}^{n}\left(\left(F\left(\frac{y_{(i+1)}^{*}-\mathcal{M}}{\delta}\right)-\hat{F}_{x}\left(y_{(i)}^{*}\right)\right)^{2} F^{\prime \delta}\left(\frac{y_{(i+1)}^{*}-\mathcal{M}}{\delta}\right)-\left(F\left(\frac{y_{(i)}^{*}-\mathcal{M}}{\delta}\right)-\hat{F}_{x}\left(y_{(i)}^{*}\right)\right)^{2} F^{\prime \delta}\left(\frac{y_{(i)}^{*}-\mathcal{M}}{\delta}\right)\right)=0
\end{array}\right\} .
$$

The estimates based on solving (11) will be denoted as $\hat{\theta}_{F}=\left(\widehat{\mathcal{M}}_{F}, \hat{\delta}_{F}\right)$. To close this section, it should be indicated that Ozturk [23] used EM algorithm to suggest a new estimation method under RSS. However, his strategy required heavy computations and time consuming causing to be omitted in this work. Another important point deserves to be mentioned is to obtain the variances estimates of the proposed estimators. 
Since we feel that deriving these variances seems not an easy mission, an approximation can be simply obtained by using bootstrap RSS by rows (BRSSR) method proposed by Modarres et al. [24] similar to what is done in Mahdizadeh and Tamandi [13].

\section{EFFICIENCY COMPARISON USING SIMULATED DATA SET}

This part exhibits the performance of the aforementioned estimation methods based on the simulated data generated through Dell and Clutter [25] model which assuming $(Y, X)$ has a standard bivariate normal distribution with a correlation coefficient $\rho$. The chosen values of $\rho$ are: $\rho=1, .80$ and . 50. To illustrate the effect of set and sample sizes, we considered four different configurations: $(k, m)=$ $(2,5),(2,10),(5,2)$ and $(5,4)$. Then we take $Y, \phi_{L}^{-1}\left(\phi_{n}(Y)\right), \phi_{G}^{-1}\left(\phi_{n}(Y)\right)$ and $\phi_{E}^{-1}\left(\phi_{n}(Y)\right)$ as the target variables, where $\phi_{n}(\cdot)$ is the $\mathrm{CDF}$ for the standard normal and $\phi_{L}^{-1}(\cdot), \phi_{G}^{-1}(\cdot)$ and $\phi_{E}^{-1}(\cdot)$ are respectively the quantile function for logistic, gumbel and exponential distributions. For each combination of $\rho, k$ and $m$, data sets are generated from the target variables based on 5,000 samples under RSS. The biases and the MSEs for $\hat{\theta}_{L}, \hat{\theta}_{T}, \hat{\theta}_{C}, \hat{\theta}_{E}, \hat{\theta}_{X}$ and $\hat{\theta}_{F}$ estimators were computed and reported as shown by Table 1.

In the context of the simulation results, the following points can be concluded:

1- For a fixed sample size, increasing the set size rather than the number of cycles has a positive effect on the biases and the MSEs of the estimators provided that the rankings are perfect done.

2- For a fixed set size increasing the sample size has a positive effect on the biases and the MSEs of the estimators regardless the quality ranking.

3- As expected, there is a touchable effect of the values of $\rho$ on the behavior of the considered estimators, as increasing the values of $\rho$ decreases the biases and MSEs of all estimators and vice versa.

4- Concerned about estimating $\mathcal{M}$, it is clear to say that the differences between the performance of the considered estimators are quite close and can be ignorable. Therefore, it does not matter which estimation method should be adopted for estimating $\mathcal{M}$.

5- On the other hand, concerned about estimating $\delta$, it is easily to see that the behavior of the estimators has various patterns. Under the perfect ranking, $\hat{\theta}_{L}$ and $\hat{\theta}_{F}$ are the best estimators with respect to the bias and the MSE criteria with a few exceptions. Whereas this superiority decreases in favor to $\hat{\theta}_{X}$ and $\hat{\theta}_{E}$ as the quality of the ranking goes to the imperfect case.

6- Although both $\hat{\theta}_{L}$ and $\hat{\theta}_{E}$ deal with the RSS items as independent order statistics, it is apparent that $\hat{\theta}_{E}$ is much less sensitive to the perfectness assumption than $\hat{\theta}_{L}$. The reason for this phenomenon is that $\hat{\theta}_{E}$ uses the fraction-of-random-rankings model which reduces the effect of violating the perfectness assumption.

7- It is also evident that $\hat{\theta}_{X}$ outperforms $\hat{\theta}_{C}$ in all the situations as long as the rankings are done either perfect or nearly perfect.

\section{EFFICIENCY COMPARISON USING EMPIRICAL DATA SET}

In what follows, we assess the performances of $\hat{\theta}_{L}, \hat{\theta}_{T}, \hat{\theta}_{C}, \hat{\theta}_{E}, \hat{\theta}_{X}$ and $\hat{\theta}_{F}$ estimators using the empirical data set known as Veterans' Administration Lung Cancer Study (WALCS) data set and is available in survival package of $\mathrm{R}$ statistical software. We will consider this data set as the target population which includes eight variables with 137 observations yet only two variables will be considered: the "karno" as the concomitant variable $X$ and the "time" as the variable of interest $Y$. These two variables are not highly correlated as their Pearson correlation coefficient is only $37.5 \%$, thus one can expect that if RSS is collected it will be nearly imperfect. In the light of the fact that Zamanzade [26] demonstrated that $Y$ follows the exponential distribution with $\delta=121.67$ using CDF based goodness of fit tests, another comparison study is performed by the following procedures. For the same values of $(k, m)$ determined in the preceding Section, 5,000 concomitant-based RSS with replacement were selected from the target population. For each sample, $\hat{\delta}_{L}, \hat{\delta}_{T}, \hat{\delta}_{C}, \hat{\delta}_{E}, \hat{\delta}_{X}$ and $\hat{\delta}_{F}$ estimators were estimated and their biases and MSEs were also obtained and plotted as presented by Figure 1. 
Table 1. Estimated biases and MSEs of the methods of estimation using the simulated data

\begin{tabular}{|c|c|c|c|c|c|c|c|c|c|c|c|c|c|c|}
\hline & & & \multicolumn{6}{|c|}{$\mathcal{M}$} & \multicolumn{6}{|c|}{$\delta$} \\
\hline & \multirow{2}{*}{$(k . m)$} & & \multicolumn{2}{|l|}{$\rho=1$} & \multicolumn{2}{|c|}{$\rho=0.80$} & \multicolumn{2}{|c|}{$\rho=0.50$} & \multicolumn{2}{|l|}{$\rho=1$} & \multicolumn{2}{|c|}{$\rho=0.80$} & \multicolumn{2}{|c|}{$\rho=0.50$} \\
\hline & & & Bias & MSE & Bias & MSE & Bias & MSE & Bias & MSE & Bias & MSE & Bias & MSE \\
\hline \multirow{24}{*}{ 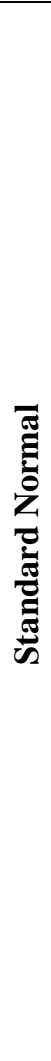 } & \multirow{6}{*}{$(2,5)$} & $\hat{\theta}_{L}$ & 0.002 & 0.065 & 0.005 & 0.081 & 0.017 & 0.088 & 0.052 & 0.045 & 0.012 & 0.056 & 0.040 & 0.071 \\
\hline & & $\hat{\theta}_{T}$ & 0.003 & 0.066 & 0.005 & 0.083 & 0.015 & 0.087 & 0.222 & 0.089 & 0.234 & 0.098 & 0.241 & 0.099 \\
\hline & & $\hat{\theta}_{C}$ & 0.005 & 0.069 & 0.005 & 0.089 & 0.010 & 0.091 & 0.018 & 0.087 & 0.035 & 0.085 & 0.051 & 0.080 \\
\hline & & $\hat{\theta}_{E}$ & 0.002 & 0.065 & 0.005 & 0.081 & 0.017 & 0.087 & 0.065 & 0.046 & 0.053 & 0.057 & 0.051 & 0.054 \\
\hline & & $\hat{\theta}_{X}$ & 0.002 & 0.052 & 0.001 & 0.076 & 0.012 & 0.085 & 0.054 & 0.044 & 0.063 & 0.057 & 0.072 & 0.054 \\
\hline & & $\widehat{\theta}_{F}$ & 0.005 & 0.053 & 0.001 & 0.085 & 0.004 & 0.091 & 0.033 & 0.048 & 0.055 & 0.085 & 0.071 & 0.092 \\
\hline & \multirow{6}{*}{$(2,10)$} & $\hat{\theta}_{L}$ & 0.004 & 0.033 & 0.006 & 0.038 & 0.001 & 0.042 & 0.038 & 0.023 & 0.003 & 0.025 & 0.083 & 0.040 \\
\hline & & $\hat{\theta}_{T}$ & 0.004 & 0.033 & 0.008 & 0.038 & 0.001 & 0.042 & 0.142 & 0.043 & 0.152 & 0.045 & 0.144 & 0.043 \\
\hline & & $\hat{\theta}_{C}$ & 0.007 & 0.036 & 0.005 & 0.042 & 0.004 & 0.046 & 0.025 & 0.043 & 0.037 & 0.042 & 0.027 & 0.042 \\
\hline & & $\hat{\theta}_{E}$ & 0.004 & 0.033 & 0.007 & 0.038 & 0.002 & 0.041 & 0.046 & 0.024 & 0.027 & 0.024 & 0.001 & 0.028 \\
\hline & & $\hat{\theta}_{X}$ & 0.004 & 0.027 & 0.004 & 0.038 & 0.002 & 0.041 & 0.046 & 0.021 & 0.049 & 0.025 & 0.037 & 0.027 \\
\hline & & $\hat{\theta}_{F}$ & 0.003 & 0.029 & 0.002 & 0.037 & 0.008 & 0.045 & 0.033 & 0.021 & 0.043 & 0.036 & 0.028 & 0.044 \\
\hline & \multirow{6}{*}{$(5,2)$} & $\hat{\theta}_{L}$ & 0.007 & 0.036 & 0.017 & 0.060 & 0.003 & 0.079 & 0.039 & 0.031 & 0.066 & 0.062 & 0.282 & 0.194 \\
\hline & & $\hat{\theta}_{T}$ & 0.006 & 0.036 & 0.017 & 0.064 & 0.003 & 0.082 & 0.210 & 0.074 & 0.238 & 0.092 & 0.244 & 0.097 \\
\hline & & $\hat{\theta}_{C}$ & 0.001 & 0.038 & 0.016 & 0.066 & 0.004 & 0.089 & 0.008 & 0.061 & 0.031 & 0.076 & 0.056 & 0.083 \\
\hline & & $\hat{\theta}_{E}$ & 0.007 & 0.031 & 0.018 & 0.06 & 0.004 & 0.079 & 0.043 & 0.031 & 0.012 & 0.051 & 0.037 & 0.064 \\
\hline & & $\hat{\theta}_{X}$ & 0.008 & 0.022 & 0.022 & 0.055 & 0.002 & 0.084 & 0.055 & 0.031 & 0.07 & 0.046 & 0.082 & 0.051 \\
\hline & & $\hat{\theta}_{F}$ & 0.004 & 0.022 & 0.021 & 0.063 & 0.001 & 0.096 & 0.032 & 0.023 & 0.048 & 0.074 & 0.070 & 0.087 \\
\hline & \multirow{6}{*}{$(5,4)$} & $\hat{\theta}_{L}$ & 0.003 & 0.018 & 0.005 & 0.028 & 0.019 & 0.043 & 0.020 & 0.016 & 0.120 & 0.045 & 0.190 & 0.346 \\
\hline & & $\hat{\theta}_{T}$ & 0.005 & 0.019 & 0.005 & 0.028 & 0.019 & 0.043 & 0.124 & 0.031 & 0.136 & 0.040 & 0.043 & 0.142 \\
\hline & & $\hat{\theta}_{C}$ & 0.001 & 0.011 & 0.004 & 0.030 & 0.022 & 0.049 & 0.004 & 0.029 & 0.020 & 0.038 & 0.040 & 0.029 \\
\hline & & $\hat{\theta}_{E}$ & 0.003 & 0.011 & 0.006 & 0.028 & 0.02 & 0.043 & 0.021 & 0.016 & 0.074 & 0.033 & 0.052 & 0.052 \\
\hline & & $\hat{\theta}_{X}$ & 0.003 & 0.017 & 0.002 & 0.025 & 0.02 & 0.044 & 0.035 & 0.014 & 0.036 & 0.024 & 0.028 & 0.041 \\
\hline & & $\hat{\theta}_{F}$ & 0.002 & 0.016 & 0.001 & 0.027 & 0.022 & 0.052 & 0.018 & 0.001 & 0.031 & 0.038 & 0.044 & 0.039 \\
\hline \multirow{24}{*}{ 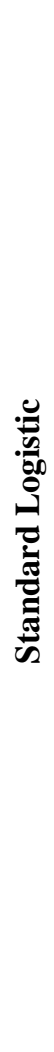 } & \multirow{6}{*}{$(2,5)$} & $\hat{\theta}_{L}$ & 0.027 & 0.220 & 0.011 & 0.243 & 0.014 & 0.271 & 0.045 & 0.056 & 0.005 & 0.072 & 0.068 & 0.103 \\
\hline & & $\hat{\theta}_{T}$ & 0.025 & 0.223 & 0.011 & 0.244 & 0.015 & 0.271 & 0.214 & 0.094 & 0.233 & 0.104 & 0.249 & 0.108 \\
\hline & & $\hat{\theta}_{C}$ & 0.024 & 0.226 & 0.012 & 0.247 & 0.018 & 0.282 & 0.002 & 0.087 & 0.028 & 0.095 & 0.052 & 0.089 \\
\hline & & $\hat{\theta}_{E}$ & 0.032 & 0.225 & 0.009 & 0.261 & 0.015 & 0.271 & 0.084 & 0.062 & 0.065 & 0.072 & 0.069 & 0.073 \\
\hline & & $\hat{\theta}_{X}$ & 0.025 & 0.198 & 0.014 & 0.233 & 0.011 & 0.272 & 0.077 & 0.059 & 0.084 & 0.073 & 0.102 & 0.075 \\
\hline & & $\hat{\theta}_{F}$ & 0.020 & 0.174 & 0.017 & 0.232 & 0.010 & 0.281 & 0.039 & 0.047 & 0.049 & 0.098 & 0.071 & 0.094 \\
\hline & & $\hat{\theta}_{L}$ & 0.007 & 0.096 & 0.006 & 0.117 & 0.020 & 0.140 & 0.024 & 0.032 & 0.028 & 0.038 & 0.123 & 0.069 \\
\hline & & $\hat{\theta}_{T}$ & 0.008 & 0.096 & 0.008 & 0.118 & 0.019 & 0.140 & 0.131 & 0.047 & 0.130 & 0.048 & 0.131 & 0.050 \\
\hline & 1210 & $\hat{\theta}_{C}$ & 0.006 & 0.096 & 0.006 & 0.121 & 0.018 & 0.142 & 0.016 & 0.048 & 0009 & 0.052 & 0.008 & 0.056 \\
\hline & & $\hat{\theta}_{E}$ & 0.006 & 0.106 & 0.009 & 0.12 & 0.023 & 0.15 & 0.041 & 0.038 & 0.017 & 0.036 & 0.011 & 0.045 \\
\hline & & $\hat{\theta}_{X}$ & 0.001 & 0.088 & 0.006 & 0.116 & 0.014 & 0.154 & 0.042 & 0.034 & 0.043 & 0.037 & 0.035 & 0.043 \\
\hline & & $\hat{\theta}_{F}$ & 0.004 & 0.077 & 0.011 & 0.110 & 0.010 & 0.141 & 0.021 & 0.024 & 0.026 & 0.045 & 0.011 & 0.057 \\
\hline & & $\hat{\theta}_{L}$ & 0.014 & 0.100 & 0.011 & 0.206 & 0.013 & 0.243 & 0.043 & 0.045 & 0.123 & 0.093 & 0.371 & 0.352 \\
\hline & & $\hat{\theta}_{T}$ & 0.12 & 0.099 & 0.011 & 0.205 & 0.015 & 0.252 & 0.213 & 0.085 & 0.213 & 0.093 & 0.243 & 0.112 \\
\hline & (5. & $\hat{\theta}_{C}$ & 0.014 & 0.099 & 0.013 & 0.209 & 0.015 & 0.263 & 0.006 & 0.069 & 0.023 & 0.093 & 0.042 & 0.123 \\
\hline & $(0,2)$ & $\hat{\theta}_{E}$ & 0.019 & 0.073 & 0.011 & 0.212 & 0.011 & 0.243 & 0.054 & 0.049 & 0.044 & 0.076 & 0.042 & 0.087 \\
\hline & & $\hat{\theta}_{X}$ & 0.019 & 0.105 & 0.023 & 0.203 & 0.009 & 0.253 & 0.081 & 0.048 & 0.068 & 0.074 & 0.092 & 0.073 \\
\hline & & $\hat{\theta}_{F}$ & 0.006 & 0.105 & 0.032 & 0.192 & 0.014 & 0.274 & 0.044 & 0.024 & 0.066 & 0.083 & 0.071 & 0.112 \\
\hline & & $\hat{\theta}_{L}$ & 0.003 & 0.046 & 0.035 & 0.090 & 0.001 & 0.119 & 0.016 & 0.021 & 0.137 & 0.062 & 0.409 & 0.254 \\
\hline & & $\hat{\theta}_{T}$ & 0.004 & 0.047 & 0.035 & 0.089 & 0.001 & 0.121 & 0.122 & 0.036 & 0.134 & 0.045 & 0.139 & 0.047 \\
\hline & $(5)$ & $\hat{\theta}_{C}$ & 0.007 & 0.048 & 0.036 & 0.092 & 0.002 & 0.123 & 0.001 & 0.034 & 0.178 & 0.044 & 0.020 & 0.047 \\
\hline & $(5,4)$ & $\hat{\theta}_{E}$ & 0.008 & 0.047 & 0.037 & 0.094 & 0.001 & 0.122 & 0.026 & 0.022 & 0.078 & 0.043 & 0.126 & 0.07 \\
\hline & & $\hat{\theta}_{X}$ & 0.007 & 0.033 & 0.03 & 0.091 & 0.001 & 0.126 & 0.048 & 0.022 & 0.052 & 0.033 & 0.053 & 0.037 \\
\hline & & $\hat{\theta}_{F}$ & 0.003 & 0.030 & 0.028 & 0.083 & 0.002 & 0.128 & 0.018 & 0.009 & 0.018 & 0.044 & 0.026 & 0.052 \\
\hline
\end{tabular}


Table 1. Continue

\begin{tabular}{|c|c|c|c|c|c|c|c|c|c|c|c|c|c|c|}
\hline & & & \multicolumn{6}{|c|}{$\mathcal{M}$} & \multicolumn{6}{|c|}{$\delta$} \\
\hline & \multirow{2}{*}{$(k . m)$} & & \multicolumn{2}{|l|}{$\rho=1$} & \multicolumn{2}{|c|}{$\rho=0.80$} & \multicolumn{2}{|c|}{$\rho=0.50$} & \multicolumn{2}{|l|}{$\rho=1$} & \multicolumn{2}{|c|}{$\rho=0.80$} & \multicolumn{2}{|c|}{$\rho=0.50$} \\
\hline & & & Bias & MSE & Bias & MSE & Bias & MSE & Bias & MSE & Bias & MSE & Bias & MSE \\
\hline \multirow{24}{*}{ 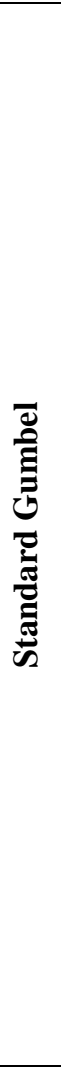 } & \multirow{6}{*}{$(2,5)$} & $\hat{\theta}_{L}$ & 0.049 & 0.089 & 0.009 & 0.084 & 0.009 & 0.109 & 0.066 & 0.059 & 0.010 & 0.060 & 0.048 & 0.096 \\
\hline & & $\hat{\theta}_{T}$ & 0.090 & 0.100 & 0.060 & 0.091 & 0.074 & 0.119 & 0.230 & 0.102 & 0.223 & 0.100 & 0.235 & 0.109 \\
\hline & & $\hat{\theta}_{C}$ & 0.045 & 0.099 & 0.016 & 0.094 & 0.036 & 0.123 & 0.016 & 0.100 & 0.015 & 0.110 & 0.031 & 0.110 \\
\hline & & $\hat{\theta}_{E}$ & 0.069 & 0.092 & 0.031 & 0.086 & 0.047 & 0.114 & 0.104 & 0.075 & 0.062 & 0.085 & 0.068 & 0.093 \\
\hline & & $\hat{\theta}_{X}$ & 0.056 & 0.069 & 0.042 & 0.08 & 0.057 & 0.115 & 0.114 & 0.059 & 0.087 & 0.077 & 0.099 & 0.099 \\
\hline & & $\hat{\theta}_{F}$ & 0.040 & 0.072 & 0.031 & 0.084 & 0.040 & 0.122 & 0.038 & 0.059 & 0.038 & 0.103 & 0.041 & 0.120 \\
\hline & \multirow{6}{*}{$(2,10)$} & $\hat{\theta}_{L}$ & 0.006 & 0.037 & 0.014 & 0.046 & 0.005 & 0.064 & 0.035 & 0.025 & 0.007 & 0.031 & 0.064 & 0.088 \\
\hline & & $\hat{\theta}_{T}$ & 0.021 & 0.038 & 0.049 & 0.049 & 0.044 & 0.055 & 0.142 & 0.045 & 0.152 & 0.050 & 0.139 & 0.044 \\
\hline & & $\hat{\theta}_{C}$ & 0.003 & 0.043 & 0.029 & 0.053 & 0.024 & 0.062 & 0.028 & 0.045 & 0.037 & 0.049 & 0.020 & 0.044 \\
\hline & & $\hat{\theta}_{E}$ & 0.006 & 0.039 & 0.028 & 0.047 & 0.023 & 0.055 & 0.041 & 0.046 & 0.031 & 0.041 & 0.006 & 0.047 \\
\hline & & $\hat{\theta}_{X}$ & 0.009 & 0.029 & 0.029 & 0.042 & 0.035 & 0.053 & 0.057 & 0.032 & 0.067 & 0.041 & 0.056 & 0.048 \\
\hline & & $\hat{\theta}_{F}$ & 0.001 & 0.030 & 0.022 & 0.046 & 0.025 & 0.055 & 0.026 & 0.022 & 0.036 & 0.045 & 0.026 & 0.046 \\
\hline & \multirow{6}{*}{$(5,2)$} & $\hat{\theta}_{L}$ & 0.007 & 0.036 & 0.006 & 0.064 & 0.044 & 0.108 & 0.050 & 0.038 & 0.069 & 0.070 & 0.299 & 0.240 \\
\hline & & $\hat{\theta}_{T}$ & 0.045 & 0.039 & 0.075 & 0.071 & 0.087 & 0.116 & 0.218 & 0.081 & 0.234 & 0.100 & 0.238 & 0.102 \\
\hline & & $\hat{\theta}_{C}$ & 0.002 & 0.038 & 0.031 & 0.073 & 0.042 & 0.114 & 0.001 & 0.068 & 0.032 & 0.095 & 0.029 & 0.091 \\
\hline & & $\hat{\theta}_{E}$ & 0.02 & 0.036 & 0.026 & 0.066 & 0.026 & 0.105 & 0.068 & 0.058 & 0.02 & 0.09 & 0.058 & 0.131 \\
\hline & & $\hat{\theta}_{X}$ & 0.024 & 0.025 & 0.047 & 0.064 & 0.071 & 0.114 & 0.11 & 0.043 & 0.101 & 0.075 & 0.086 & 0.099 \\
\hline & & $\hat{\theta}_{F}$ & 0.010 & 0.023 & 0.034 & 0.070 & 0.026 & 0.118 & 0.034 & 0.025 & 0.065 & 0.081 & 0.035 & 0.098 \\
\hline & \multirow{6}{*}{$(5,4)$} & $\hat{\theta}_{L}$ & 0.007 & 0.020 & 0.025 & 0.034 & 0.082 & 0.056 & 0.027 & 0.018 & 0.013 & 0.045 & 0.341 & 0.196 \\
\hline & & $\hat{\theta}_{T}$ & 0.027 & 0.022 & 0.029 & 0.036 & 0.039 & 0.052 & 0.129 & 0.034 & 0.017 & 0.043 & 0.160 & 0.049 \\
\hline & & $\hat{\theta}_{C}$ & 0.004 & 0.022 & 0.008 & 0.039 & 0.015 & 0.054 & 0.011 & 0.033 & 0.014 & 0.041 & 0.043 & 0.045 \\
\hline & & $\hat{\theta}_{E}$ & 0.013 & 0.02 & 0.011 & 0.036 & 0.013 & 0.051 & 0.032 & 0.028 & 0.071 & 0.047 & 0.102 & 0.078 \\
\hline & & $\hat{\theta}_{X}$ & 0.018 & 0.012 & 0.021 & 0.034 & 0.026 & 0.048 & 0.071 & 0.019 & 0.066 & 0.033 & 0.076 & 0.045 \\
\hline & & $\hat{\theta}_{F}$ & 0.006 & 0.011 & 0.013 & 0.034 & 0.014 & 0.050 & 0.023 & 0.010 & 0.022 & 0.038 & 0.047 & 0.052 \\
\hline \multirow{24}{*}{ 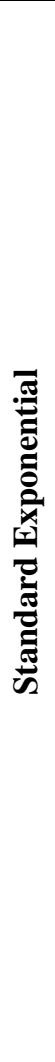 } & \multirow{6}{*}{$(2,5)$} & $\hat{\theta}_{L}$ & --- & --- & --- & --- & --- & --- & 0.074 & 0.101 & 0.057 & 0.130 & 0.023 & 0.112 \\
\hline & & $\hat{\theta}_{T}$ & --- & --- & --- & --- & --- & --- & 0.187 & 0.156 & 0.197 & 0.217 & 0.201 & 0.207 \\
\hline & & $\hat{\theta}_{C}$ & --- & --- & --- & --- & --- & --- & 0.060 & 0.122 & 0.095 & 0.251 & 0.088 & 0.187 \\
\hline & & $\hat{\theta}_{E}$ & --- & --- & --- & --- & --- & --- & 0.084 & 0.105 & 0.082 & 0.139 & 0.078 & 0.135 \\
\hline & & $\hat{\theta}_{X}$ & --- & --- & --- & --- & --- & --- & 0.09 & 0.085 & 0.097 & 0.129 & 0.1 & 0.141 \\
\hline & & $\hat{\theta}_{F}$ & --- & --- & --- & --- & --- & --- & 0.048 & 0.087 & 0.083 & 0.172 & 0.098 & 0.215 \\
\hline & & $\hat{\theta}_{L}$ & --- & --- & --- & --- & --- & --- & 0.037 & 0.044 & 0.016 & 0.047 & 0.009 & 0.056 \\
\hline & & $\hat{\theta}_{T}$ & --- & --- & --- & --- & --- & --- & 0.105 & 0.065 & 0.111 & 0.070 & 0.116 & 0.085 \\
\hline & $(2,10)$ & $\hat{\theta}_{C}$ & --- & --- & --- & --- & --- & --- & 0.031 & 0.054 & 0.031 & 0.058 & 0.052 & 0.084 \\
\hline & $(2,10)$ & $\hat{\theta}_{E}$ & --- & --- & --- & --- & --- & --- & 0.04 & 0.045 & 0.029 & 0.049 & 0.031 & 0.061 \\
\hline & & $\hat{\theta}_{X}$ & --- & --- & --- & --- & --- & --- & 0.048 & 0.035 & 0.051 & 0.046 & 0.056 & 0.067 \\
\hline & & $\hat{\theta}_{F}$ & --- & --- & --- & --- & --- & --- & 0.029 & 0.039 & 0.034 & 0.051 & 0.058 & 0.088 \\
\hline & & $\hat{\theta}_{L}$ & --- & --- & --- & --- & --- & --- & 0.043 & 0.043 & 0.019 & 0.067 & 0.015 & 0.099 \\
\hline & & $\hat{\theta}_{T}$ & --- & --- & --- & --- & --- & --- & 0.152 & 0.077 & 0.185 & 0.134 & 0.221 & 0.204 \\
\hline & $(5,2)$ & $\hat{\theta}_{C}$ & --- & --- & --- & --- & --- & --- & 0.044 & 0.068 & 0.070 & 0.116 & 0.095 & 0.173 \\
\hline & $(0,2)$ & $\hat{\theta}_{E}$ & --- & --- & --- & --- & --- & --- & 0.045 & 0.043 & 0.038 & 0.073 & 0.064 & 0.124 \\
\hline & & $\hat{\theta}_{X}$ & --- & --- & --- & --- & --- & --- & 0.066 & 0.031 & 0.093 & 0.086 & 0.132 & 0.151 \\
\hline & & $\hat{\theta}_{F}$ & --- & --- & --- & --- & --- & --- & 0.028 & 0.028 & 0.064 & 0.109 & 0.112 & 0.203 \\
\hline & & $\hat{\theta}_{L}$ & --- & --- & --- & --- & --- & --- & 0.024 & 0.019 & 0.026 & 0.032 & 0.098 & 0.046 \\
\hline & & $\hat{\theta}_{T}$ & --- & --- & --- & --- & --- & --- & 0.091 & 0.032 & 0.094 & 0.050 & 0.081 & 0.060 \\
\hline & $(5,4)$ & $\hat{\theta}_{C}$ & --- & --- & --- & --- & --- & --- & 0.015 & 0.021 & 0.018 & 0.045 & 0.008 & 0.060 \\
\hline & $(0,4)$ & $\hat{\theta}_{E}$ & -- & --- & --- & --- & --- & --- & 0.024 & 0.018 & 0.012 & 0.032 & 0.032 & 0.044 \\
\hline & & $\hat{\theta}_{X}$ & --- & --- & --- & --- & --- & --- & 0.0947 & 0.014 & 0.044 & 0.033 & 0.023 & 0.047 \\
\hline & & $\hat{\theta}_{F}$ & --- & --- & --- & --- & --- & --- & 0.017 & 0.014 & 0.027 & 0.041 & 0.006 & 0.057 \\
\hline
\end{tabular}


One can easily deduce that there is a high agreement between the results shown by Figure 1 and those reported by Table 1. As due to the weakness of the relation between $X$ and $Y$, for a fixed sample size, increasing the cycle size rather than the set size has a better effect on all the considered estimators particularly $\hat{\delta}_{L}$ which is uniformly the worst estimator in terms of both the bias and MSE criteria. In contradiction, $\hat{\delta}_{E}$ and $\hat{\delta}_{X}$ have satisfied behavior particularly at the small sample sizes yet $\hat{\delta}_{F}$ has relatively high bias in almost cases. In spite of the superiority of $\hat{\delta}_{T}$ in terms of bias criterion, it has a slight higher MSE. Finally it should be informed that all simulation studies in this work are programmed using $\mathrm{R}$ statistical software and available under request from the second author.
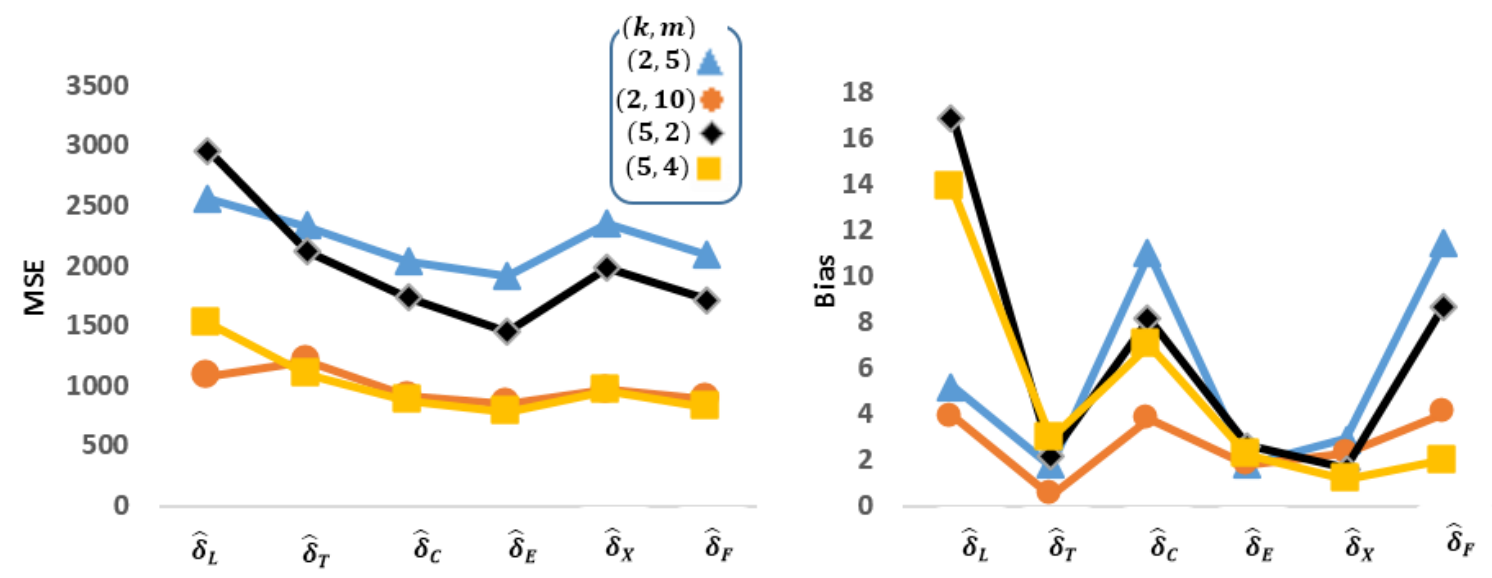

Figure 1. Estimated biases and MSEs of the methods of estimation using the empirical data. This figure appears in color in the electronic version of this article

\section{CONCLUSION}

In this study, we provide three novel methods for estimating location-scale parameters under RSS namely Iterative Moments, Modified Moments and Modified Cramér-Von Mises. The first method is based on EM algorithm, while the second and the third methods are derived under existing of the concomitant basedinformation. In the view of bias and MSE, the numerical findings recommended that the proposed methods are more efficient than their competitors in almost cases and they are relatively less sensitive to the perfectness assumption. Although we confine our attention on the location-scale family, the proposed methods can be straightforwardly extended to the non location-scale family either by directly applying the proposed methods or transforming to a distribution belongs to the location-scale family. For instance, if $Y$ follows a Weibull distribution then $\{-\log (Y)\}$ follows a Gumbel distribution (see Cabana and Quiroz [27]). Recently, Ozturk et al. [28] constructed a Rao-Blackwellized version of maximum likelihood estimators (MLE) and best linear unbiased estimators (BLUE) under judgment post stratified (JPS) samples, therefore it might be a good potential point for future works to extend our proposed methods to JPS samples.

\section{ACKNOWLEDGMENTS}

The authors are grateful to anonymous referees and an editor for their valuable comments and suggestions which lead to this improved version.

\section{CONFLICTS OF INTEREST}

No conflict of interest was declared by the authors.

\section{REFERENCES}

[1] Mittelhammer, R.C., "Mathematical Statistics for Economics and Business", Springer Science + Business Media. , (2013). 
[2] McIntyre, G.A., "A method for unbiased selective sampling using ranked set sampling", Australian Journal of Agricultural Research, 3: 385-390, (1952).

[3] Abu-Dayyeh, W., Assrhani, A., Ibrahim, K., "Estimation of the shape and scale parameters of Pareto distribution using ranked set sampling", Statistical Papers, 54: 207-225, (2013).

[4] Sarikavanij, S., Kasala, S., Sinha, B.K. and Tiensuwan, M. "Estimation of location and scale parameters in two-parameter exponential distribution based on ranked set sampling", Communications in Statistics-Simulation and Computation, 43(1): 132-141. (2014).

[5] Hassan, A., "Maximum likelihood and Bayes estimators of the unknown parameters for exponentiated exponential distribution using ranked set sampling", International Journal of Engineering Research and Applications, 3(1): 720-725, (2013).

[6] Yousef, O. and Al-Subh, S.A., "Estimation of Gumbel parameters under ranked set sampling", Journal of Modern Applied Statistical Methods, 13(2): 432-443, (2014).

[7] Dey, S. Salehi, M. Ahmadi, J. "Rayleigh distribution revisited via ranked set sampling", Metron, 75(1): 69-85, (2017).

[8] Arslan, G. and Ozturk, O., "Parametric inference based on partially rank ordered set samples", Journal of the Indian Statistical Association, 51 (1): 1-24, (2013).

[9] Rajagopalan, M and Dhanavanthan, P., "Statistical inference. PHI Learning Private Limited", (2012).

[10] Stokes, S.L., "Parametric ranked set sampling", Annals of the Institute of Statistical Mathematics, 47: 465-482, (1995).

[11] Macdonald, P., "An estimation procedure for mixtures of distribution”, Journal of the Royal Statistics Society. B (Methodol.), 33: 326-329, (1971).

[12] Ozturk, O. and Hettmansperger, T. P., "Generalised weighted Cramér-von Mises distance estimators", Biometrika, 84(2): 283-294., (1997).

[13] Mahdizadeha, M. and Tamandi, M., "A new approach to parameter estimation in ranked set sampling”, International Journal of Statistics \& Economics, 17(1): 40-49, (2016).

[14] Cheng, R. and Amin, N., "Estimating parameters in continuous univariate distributions with a shifted origin", Journal of the Royal Statistical Society. Series B ,.45: 394 -403, (1983).

[15] Frey, J. Ozturk, O. and Deshpande, J.V., "Nonparametric tests for perfect judgment rankings", Journal of the American Statistical Association, 102(478): 708-717, (2007).

[16] Frey, J. and Zhang, Y., "An algorithm with applications in ranked-set sampling”, Journal Statistical Computation Simulation; 88(3): 471-481, (2018).

[17] Ashour, S. and Abdalluh, M., "New Distribution Function Estimation and Perfectness Testing under Ranked Set Sampling", To appear in Journal of Modern Applied Statistical Methods, (2018).

[18] Wu, C., "On the Convergence Properties of the EM Algorithm", The Annals of Statistics, 11(1): 95103, (1983).

[19] Zamanzade, E. and Mohammadi, M., "Some modified mean estimator in ranked set sampling using a covariate", Journal of Statistical Theory and Applications, 15(2): 142-152, (2016). 
[20] Ozturk, O., "Statistical inference under a stochastic ordering constraint in ranked set sampling", Nonparametric Statistics, 19: 131-144, (2007).

[21] Zamanzade, E. and Mahdizadeh, M., "Distribution function estimation using concomitant-based ranked set sampling", Hacettepe Journal of Mathematics and Statistics, 47(3): 755-761, (2018).

[22] Ashour, S. and Abdalluh, M., "Estimation of Distribution Function based on Ranked Set Sampling: Missing Data Approach", To appear in Thailand Statistician, (2018).

[23] Ozturk, O., "Parametric estimation of location and scale parameters in ranked set sampling", Journal of Statistical Planning and Inference, 141(4): 1616-1622, (2011).

[24] Modarres, R., Terrence, P.H., and Zheng, G., "Resampling methods for ranked set samples". Computational Statistics and Data Analysis, 51: 1039-1050, (2006).

[25] Dell, TR. and Clutter, J.L., "Ranked set sampling theory with order statistics background", Biometrics 28(2): 545-555, (1972).

[26] Zamanzade, E., "EDF-based tests of exponentiality in pair ranked set sampling", Statistical Papers, $1-19,(2018)$.

[27] Cabana, A. and Quiroz, A.J., "Using the empirical moment generating function in testing for the Weibull and the type I extreme value distributions", Test, 14(2): 417-431, (2005).

[28] Ozturk, O. Sultan, K. S. and Moshref, M. E., "Parametric inference based on judgment post stratified samples", Journal of the Korean Statistical Society, 47(1): 24-31, (2018). 